\title{
Prevalence of Chronic Kidney Disease in Relatives of Chronic Hemodialysis Patients
}

\author{
Said Sayed Ahmed Khamis, Rawhia Hassan El Edel, Ahmed Mohamed Zahran, \\ Mohamed Ali Ibrahim*, Khaled Mohamed Amin El Zorkany \\ Internal Medicine Department, Faculty of Medicine, Menoufia University, Shebeen Elkom, Menoufia, Egypt \\ Email: *mido_01698@yahoo.com
}

How to cite this paper: Khamis, S.S.A., El Edel, R.H., Zahran, A.M., Ibrahim, M.A., El Zorkany, K.M.A. (2020) Prevalence of Chronic Kidney Disease in Relatives of Chronic Hemodialysis Patients. Open Journal of Nephrology, 10, 274-281.

https://doi.org/10.4236/ojneph.2020.104027

Received: August 25, 2020

Accepted: October 13, 2020

Published: October 16, 2020

Copyright $\odot 2020$ by author(s) and Scientific Research Publishing Inc. This work is licensed under the Creative Commons Attribution International License (CC BY 4.0).

http://creativecommons.org/licenses/by/4.0/

\begin{abstract}
Objectives: To estimate the prevalence of chronic kidney disease (CKD) in first and second degree relatives of Hemodialysis patients. Background: Early detection and intervention of chronic kidney disease (CKD) may prevent or delay the progression and achieve improved patient outcomes. Family members of end-stage renal disease (ESRD) patients are considered as a high-risk population for CKD. The aim of this work is to screen and estimate the prevalence of chronic kidney disease in first and second degree relatives of hemodialysis patients. Methods: This is an observational prospective study carried out in Nephrology Unit, Internal Medicine Department, Menoufia University Hospital during January 2019 to January 2020. First and second degree relatives of ESRD were included. Relatives with known CKD risk factors or relative to ESRD with known hereditary disease were excluded. Serum creatinine and estimated eGFR, albumin creatinine ratio (ACR) and abdominal ultrasound were done twice with 3 months apart to screen for CKD. Results: 321 persons from first and second degree relatives of chronic hemodialysis patients were included. CKD prevalence was 5.6\% Comparison between CKD group and non CKD showed no statistically significant difference as regard age, Gender, Smoking, BMI, and degree of relatives. There were highly significant difference between the studied groups as regard serum creatinine, bloodurea, eGFR and ACR. Relatives of ESRD patients of unknown etiology showed highly significant difference to develop CKD (44.4\%) compared to non CKD group $11.5 \%$ with $\mathrm{P}$ value $<0.0001$. Conclusions: Prevalence of CKD in relatives-without any CKD risk factors-to ESRD was 5.6\%. Family members of ESRD should be screened for CKD, especially relatives to ESRD of unknown etiology.
\end{abstract}

\section{Keywords}

Chronic Kidney Disease, Family Members, Prevalence, End Stage Renal 
Disease

\section{Introduction}

Chronic kidney disease (CKD) is a worldwide public health problem, with increasing prevalence and lethal adverse outcomes like progressive loss of kidney function, cardiovascular disease, and premature death [1]. CKD emerge from a large number of heterogeneous disease pathways that change the function and structure of the kidney irreversibly, over months or years. The diagnosis of CKD rests on building a chronic decrease in kidney function and structural kidney damage [2].

The major risk factors for CKD include older age, hypertension, diabetes mellitus, obesity and family history of CKD [3]. Early detection and intervention for these high-risk populations may prevent or delay the progression of CKD and achieve improved patient outcomes.

Early detection and intervention for these high-risk populations may prevent or delay the progression of CKD and achieve improved patient outcomes.

Family members of end-stage renal disease (ESRD) patients are considered as a high risk population for ESRD or CKD [4]. Freedman et al. found that $20 \%$ of dialysis patients treated in Georgia, North Carolina, and South Carolina reported having first or second degree relatives with ESRD [5]. Jurkovitz et al. reported that $49.3 \%$ of family members of ESRD patients have undetected kidney disease and 13.9\% had creatinine clearance less than $60 \mathrm{~mL} / \mathrm{min}$ [6].

A more recent study found that the prevalence of CKD in relatives of hemodialysis patients was $15.8 \%$. However, high variability in the prevalence may have been caused by differences in ethnicities and screening methods. Furthermore, the risk factors associated with CKD in this population need to be identified [7].

Current recommendations by Kidney Outcomes Quality Initiative (KDOQI) and National Institute for Health Excellence (NICE) are to use serum creatinine concentration to estimate GFR (eGFR) and transform it using the Chronic Kidney Disease Epidemiology Collaboration (CKD-EPI) equation [8]. The presence of a first degree or second degree relative with ESRD was reported to increase the risk of developing ESRD by 3 to 5 fold in Americans [9]. In addition, subsequent studies revealed that a significant proportion of relatives of CKD patients had clinically "silent" kidney diseases, which was manifested as asymptomatic proteinuria and/or microscopic hematuria [10]. The reported prevalence of CKD in relatives of CKD patients ranged from $14 \%$ to $30 \%$. These findings granted support to the need for screening in these individuals that may carry higher risks of developing CKD compared with the general population [11]. Since there is a limited study that evaluates the risk of CKD in close relatives of patients with earlier stages of CKD, the Hong Kong Society of Nephrology em- 
barked on the present study to screen first degree relatives of CKD patients for the prevalence and risk factors for developing CKD in Hong Kong [12].

Hong Kong study revealed that $23.1 \%, 25.9 \%$ and $4.4 \%$ of participants had proteinuria (urine protein $\geq 1+$ ), haematuria (urine $\mathrm{RBC} \geq 1+$ ) and glycosuria (urine glucose $\geq 1+$ ), respectively. In comparison with other previous study that screened silent kidney disease in asymptomatic individuals in Hong Kong, Hong Kong study reported a significantly higher prevalence of proteinuria $(23.1 \%$ vs. $3.0 \%, P<0.0001)$, haematuria $(25.9 \%$ vs. $17.0 \%, P<0.0001)$ and glycosuria (4.4\% vs. $1.9 \%, P=0.001)$, respectively [13].

Our goal of this study to estimate the prevalence of chronic kidney disease $(\mathrm{CKD})$ in first and second degree relatives of Hemodialysis patients.

\section{Patient and Methods}

This is an observational prospective study carried out in Nephrology Unit, Internal Medicine Department, Menoufia University Hospital during January 2019 to January 2020 This study followed the ethical standards of our institute and approved by ethical committee of Menoufia University Hospital. All subjects were given informed consent.

This study included (321) person from first and second degree relative of ESRD patients. All ESRD patients on Menoufia University Hospital and Munshaat Sultan dialysis unit were asked to send their first and second degree relatives to check for CKD.

All Participants included were first and second degree relatives to ESRD patients and above 18 years old. The following subjects were excluded; relatives of ESRD patients due to hereditary disorder (APCKD, Alport syndrome), relatives Who have diabetes mellitus, hypertension, cardiovascular disease, chronic liver disease or any risk factor for CKD, persons above 65 years old and persons with acute kidney injury.

All participants were subjected to the following: History taking and clinical examination to exclude any risk factor for CKD. Serum creatinine was done and eGFR was estimated by CKD EPI equation [8]. ACR was measured using mid-stream morning urine sample. Abdominal ultrasound was done to screen for any kidney abnormality. Investigations were repeated after 3 months to confirm CKD. CKD was diagnosed according to KDIGO criteria [14].

$\mathrm{CKD}$ is defined as presence of kidney damage or decreased glomerular filtration rate less than $60 \mathrm{ml} / \mathrm{min} / 1.7 \mathrm{~m}^{2}$ for 3 months or more, irrespective of cause [14].

\section{Statistical Analysis}

Data were collected and analysed using Statistical Package for Social Sciences program (SPSS) for Windows version 22.0 (SPSS Inc., Chicago, IL, USA) and Microsoft excel sheet. Numerical data were presented as mean and standard deviation (SD) and Categorical data were presented as number and percentage. 
Chi-square $\left(\chi^{2}\right)$ was used to compare two groups of categorical variables whereas the student's t-test or Mann-Whitney test were used to compare two groups of quantitative variables as appropriate. $\mathrm{Z}$ test was used to compare two proportions.

$P$ value $<0.05$ was considered statistically significant.

\section{Results}

During the study period, 377 direct relatives from 173 ESRD patients were invited to participate into study. A total of 355 relatives from 152 families responded. Among these 355 individuals, 34 of them were excluded ( 2 were < 18 years, 7 had a known history of liver disease, 25 had a known history of hypertension and/or diabetes).

As a result, a total of 321 individuals from 152 families were included in the final analysis.

This study was carried out in Menoufia University hospital on 321 subjects 181 (56.4\%) were male and 140 (43.6\%) were female. All subjects were first and second degree relatives to ESRD patients.

Our results demonstrated that the prevalence of CKD among the studied group was 18 patients (5.6\%) (Figure 1). All participants were divided into two groups: Group 1; Normal individuals and group 2 individuals with CKD.

The mean age of the All participants was (33) year: group (1) $33.01 \pm 9.6$, group (2) $34.28 \pm 7.03$ there was no statistically significant difference between the studied groups as regard age our study includes 181 (56.4\%) of the studied patients were males and 140 (43.6\%) were females: in Group (1) (169 (56.1\%) were males \& 134 (43.9\%) were females), in Group (2) (12 (64.2\%) were males \& $6(35.8 \%)$ were female) There was no statistically significant difference between

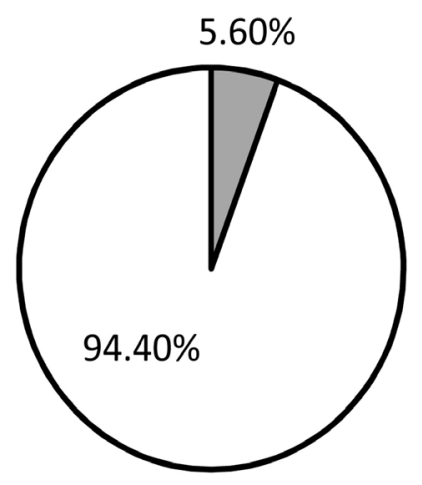

\section{口CKD $\square$ Non CKD}

Figure 1. Prevalence of CKD in relatives of chronic hemodialysis patient. CKD: Chronic kidney disease, ESRD: End stage renal disease. 
the studied groups as regard gender.

As regard Relatives: 241 (75.1\%) of the studied patients were first degree and 80 (24.9\%) were second degree: in group (1) (228 were first degree \& 75 were second degree), in group (2) (13 were first degree $\& 5$ were second degree) there was no statistically significant difference between the studied groups as regard degree of relatives. there was no statistically significant difference between the studied groups as regard BMI, Blood pressure, smoking (Table 1).

As regard Biochemical parameters: There were high significant difference between the three studied groups as regard (S. creatinine, s. urea, eGFR, ACR, urine analysis) $\mathrm{P}$ value $<0.001$ (Table 1 ).

$44 \%$ of CKD participants were relatives to patients with unknown etiology of ESRD which was highly significant when compared to non CKD participants of unknown etiology (11.5\%) (Table 2).

In our study CKD group (18) persons show 16 person (88.7\%) have proteinuria, 13 person (72\%) have GFR less than $60 \mathrm{ml} / \mathrm{min} / 1.7 \mathrm{~m}^{2}$.

\section{Discussion}

$\mathrm{CKD}$ is defined by indicators of kidney damage-imaging or proteinuria (commonly using albumin to creatinine ratio, ACR) - and decreased renal function (below thresholds of GFR estimated from serum creatinine concentration) [14].

Table 1. Comparison of the studied groups regarding demographic data.

\begin{tabular}{|c|c|c|c|c|}
\hline Variables & $\begin{array}{c}\text { Group 1 } \\
\mathrm{N}=303 \\
\text { Mean } \pm \mathrm{SD}\end{array}$ & $\begin{array}{c}\text { Group } 2 \\
N=18 \\
\text { Mean } \pm \text { SD }\end{array}$ & $\begin{array}{c}\text { Test of } \\
\text { significance }\end{array}$ & $P$ value \\
\hline Age & $33.01 \pm 9.6$ & $34.28 \pm 7.03$ & $0.5^{\star}$ & 0.5 \\
\hline \multicolumn{5}{|l|}{ Gender } \\
\hline Male (N/\%) & $169(56.1)$ & 1264.2 & $0.8^{\#}$ & 0.4 \\
\hline Female (N/\%) & $134(43.9)$ & 635.8 & & \\
\hline \multicolumn{5}{|l|}{ Smoking } \\
\hline Yes (N/\%) & $98(32.3)$ & $5(27.8)$ & $0.9^{\#}$ & 0.1 \\
\hline No (N/\%) & $205(67.7)$ & $13(72.2)$ & & \\
\hline BMI & $27.3 \pm 1.9$ & $26.5 \pm 2.02$ & $1.7^{*}$ & 0.07 \\
\hline SBP & $108.32 \pm 10.1$ & $110 \pm 9.1$ & $0.6^{*}$ & 0.4 \\
\hline DBP & $70.17 \pm 7.1$ & $72.2 \pm 4.2$ & $1.2^{\star}$ & 0.2 \\
\hline \multicolumn{5}{|l|}{ Relative } \\
\hline $1^{\text {st }}$ degree $(\mathrm{N} / \%)$ & $228(75.2)$ & $13(72.2)$ & $0.08^{\#}$ & 0.7 \\
\hline $2^{\text {nd }}$ degree $(\mathrm{N} / \%)$ & $75(24.8)$ & $5(27.8)$ & & \\
\hline Creatinine & $0.9 \pm 0.1$ & $2.4 \pm 1.2$ & $19.5^{*}$ & $<0.001$ \\
\hline eGFR & $111.3 \pm 23.2$ & $52.4 \pm 38.4$ & $10.01^{*}$ & $<0.001$ \\
\hline Urea & $28.4 \pm 5.3$ & $88.4 \pm 52.9$ & $18.3^{*}$ & $<0.001$ \\
\hline ACR & $6.14 \pm 4.4$ & $783.4 \pm 743.5$ & $-6.49^{\$}$ & $<0.001$ \\
\hline
\end{tabular}

*: Student $t$ test, " : Chi square. ${ }^{\$}$ : Mann-Whitney test, N: Number, SD: Standard Deviation, BMI: Body mass index, SBP: Systolic blood pressure, DBP: Diastolic blood pressure, eGFR: Estimated glomerular filtration rate, ACR: Albumin creatinine ratio. 
Table 2. Comparison of the studied groups regarding etiology of ESRD.

\begin{tabular}{ccccc}
\hline Variables & $\begin{array}{c}\text { Group 1 } \\
\mathrm{N}=303\end{array}$ & $\begin{array}{c}\text { Group 2 } \\
\mathrm{N}=18 \\
\text { No }(\%)\end{array}$ & Z test & P value \\
\hline Diabetes mellitus & $118(38.9)$ & $3(16.7)$ & 3.5 & 0.05 \\
Hypertension & $84(27.7)$ & $2(11.1)$ & 2.3 & 0.1 \\
Obstructive uropathy & $18(5.9)$ & $1(5.5)$ & 0.004 & 0.9 \\
Glomerulopathy & $48(15.8)$ & $4(22.2)$ & 0.5 & 0.4 \\
Unknown & $35(11.5)$ & $8(44.4)$ & 15.8 & $<0.0006$ \\
\hline
\end{tabular}

Family history of CKD was also considered targets for screening. In fact, familial clustering of ESRD has been increasingly recognized [15]. The presence of a first degree or second degree relative with ESRD was reported to increase the risk of developing ESRD by 3 - 5 folds in Americans [9].

This study includes (321) person from first and second degree relative of ESRD patients. Who met the inclusion criteria at the time of the study. Though this number is not much large as compared to international screening analysis, it still gives us direction toward the future strategies, family members of ESRD patients were recruited for CKD screening. A medical history, measurements of $\mathrm{BP}$, serum glucose, serum creatinine, and urinalysis, ACR were obtained at community screening sites. In our study of 321 relatives: 181 (56.4\%) of the studied patients were males and $140(43.6 \%)$ were females, $241(75.1 \%)$ of the studied patients were first degree and 80 (24.9\%) were second degree.

Comparing of the two studied groups regarding demographic data did not demonstrate any significant difference while the laboratory data showed highly significant difference regarding serum creatinine, blood urea, eGFR and urine albumin creatinine ratio.

Comparison between studied groupsas regard Aetiology of ESRD in relation to relatives: There were highly significant difference between the studied groups as regard aetiology of ESRD in relatives due to unknown cause ( $p$ value < 0.0001). There were no significant difference between the studied groups as regard aetiology of ESRD in relatives due to (DM, HTN, O.U, GN).

Our study show (5.6\%) 18 presons have CKD, (72.2\%) 13 person from first degree and (27.8\%) 5 persons from second degree relatives.

In another study included 221 family members screened between 1999 and 2001 in Georgia demonstrated that $13.9 \%$ had an estimated creatinine clearance (Ccr) $<60 \mathrm{ml} / \mathrm{min}$ [16]. This high prevalence rate compared to our results may be attributed to that inclusion of diabetic and hypertensive persons in their work while they were excluded in our study, similar to our results Kong X. et al. showed that $8 \%$ of 200 participants relative to ESRD patients had increased serum creatinine [12]. In our study, we identified that relatives to ESRD with unknown aetiology showed higher prevalence of CKD $44.4 \%$ which may indicate underlying unknown hereditary disease. We could not identify any work to as- 
sess the prevalence of CKD in relatives of ESRD with unknown aetiology.

This study has the limitation of small sample size and it was cross sectional observational study as we did identify the etiology of CKD in relatives as most of them were sent for follow up in outpatient clinics.

Our study carried the advantage of being the first to assess the prevalence of CKD in relatives to ESRD patients without any known risk factors for CKD. Also, we showed that relatives to ESRD patients with unknown ethology are at high risk and should be Screened and thoroughly investigated for CKD.

\section{Conclusion}

Prevalence of CKD in relatives of chronic hemodialysis patients was $5.6 \%$. Screening of the family members of ESRD patients is crucial for early detection and appropriate intervention. Family members of ESRD due to unknown cause showed higher prevalence of CKD.

\section{Conflicts of Interest}

The authors declare no conflicts of interest regarding the publication of this paper.

\section{References}

[1] Freethi, R., Velayutha Raj, A., Ponniraivan, K., Rasheed Khan, M., Sundhararajan, A. and Venkatesan (2016) Study of Serum Levels of Calcium, Phosphorus and Alkaline Phosphatase in Chronic Kidney Disease. International Journal of Medical Research \& Health Sciences, 5, 49-56.

[2] Levey, A.S., Becker, C. and Inker, L.A. (2015) Glomerular Filtration Rate and Albuminuria for Detection and Staging of Acute and Chronic Kidney Disease in Adults: A Systematic Review. JAMA, 313, 837-846. https://doi.org/10.1001/jama.2015.0602

[3] Taal, M.W. and Brenner, B.M. (2006) Predicting Initiation and Progression of Chronic Kidney Disease: Developing Renal Risk Scores. Kidney International, 70, 1694-1705. https://doi.org/10.1038/sj.ki.5001794

[4] Freedman, B.I., Spray, B.J., Tuttle, A.B. and Buckalew Jr., V.M. (1993) The Familial Risk of End-Stage Renal Disease in African Americans. American Journal of Kidney Diseases, 21, 387-393. https://doi.org/10.1016/S0272-6386(12)80266-6

[5] Freedman, B.I., Soucie, J.M. and McClellan, W.M. (1997) Family History of End-Stage Renal Disease among Incident Dialysis Patients. Journals of the American Society of Nephrology, 8, 1942-1945.

[6] Jurkovitz, C., Franch, H., Shoham, D., Bellenger, J. and McClellan, W. (2002) Family Members of Patients Treated for ESRD Have High Rates of Undetected Kidney Disease American Journal of Kidney Diseases, 40, 1173-1178. https://doi.org/10.1053/ajkd.2002.36866

[7] Tsai, J.C., Chen, S.C., Hwang, S.J., Chang, J.M., Lin, M.Y. and Chen, H.C. (2010) Prevalence and Risk Factors for CKD in Spouses and Relatives of Hemodialysis $\mathrm{Pa}$ tients. American Journal of Kidney Diseases, 55, 856-866. https://doi.org/10.1053/j.ajkd.2009.12.021

[8] Levey, A.S., Stevens, L.A., Schmid, C.H., Zhang, Y.L., Castro, A.F., Feldman, H.I., et 
al. (2009) A New Equation to Estimate Glomerular Filtration Rate. Annals of Internal Medicine, 150, 604-612.

https://doi.org/10.7326/0003-4819-150-9-200905050-00006

[9] Raji, Y.R., Mabayoje, M.O., Bello, B.T. and Amira, C.O. (2018) Albuminuria and Reduced Estimated Glomerular Filtration Rate among First-Degree Relatives of patients with Chronic Kidney Disease in Lagos, Southwest Nigeria. Indian Journal of Nephrology, 28, 21-27. https://doi.org/10.4103/ijn.IJN_225_16

[10] Webster, A.C., Nagler, E.V., Morton, R.L. and Masson, P. (2017) Chronic Kidney Disease. The Lancet, 389, 1238-1252. https://doi.org/10.1016/S0140-6736(16)32064-5

[11] Li, P.K., Ng, J.K., Cheng, Y.L., Kwan, T.H., Leung, C.B., Lau, M.F., et al. (2017) Relatives in Silent Kidney Disease Screening (RISKS) Study: A Chinese Cohort Study. Nephrology, 22, 35-42. https://doi.org/10.1111/nep.13148

[12] Kong, X.L., Liu, L., Zuo, L., Yuan, P., Li, Z.X., Li, W.G., et al. (2013) Association between Family Members of Dialysis Patients and Chronic Kidney Disease: A Multicenter Study in China. BMC Nephrology, 14, Article No.19.

https://doi.org/10.1186/1471-2369-14-19

[13] Li, P.K.T., Kwan, B.C., Leung, C.B., et al. (2005) Prevalence of silent kidney disease in Hong Kong: The Screening for Hong Kong Asymptomatic Renal Population and Evaluation (SHARE) Program. Kidney International, 67, S36-S40. https://doi.org/10.1111/j.1523-1755.2005.09410.x

[14] National Kidney Foundation (2002) KDOQI Chronic Kidney Disease: Evaluation, Classification, and Stratification Kidney Disease Outcome Quality Initiative. American Journal of Kidney Diseases, 39, S1-S266.

[15] Galbraith, L., Hemmelgarn, B., Manns, B., Samuel, S., Kappel, J., Valk, N., et al. (2016) The Association between Individual Counselling and Health Behaviour Change: The See Kidney Disease (SeeKD) Targeted Screening Programme for Chronic Kidney Disease. Canadian Journal of Kidney Health and Disease, 3, 35. https://doi.org/10.1186/s40697-016-0127-4

[16] Mital, S., Kher, V., Gulati, S., Agarwal, L.K. and Arora, P. (1997) Chronic renal failure in India. Renal Failure, 19, 763-770.

https://doi.org/10.3109/08860229709037216 